\title{
Thymic Neuroendocrine Tumour Results Cushing's Syndrome
}

\author{
Taher Manzary ${ }^{1}$, Amir Teimouri Dereshgi ${ }^{2}$, Vahideh Sadra ${ }^{1}$, Ali Jamshidi Fard ${ }^{1}$, \\ Leila Teimouri Dereshgi ${ }^{3}$, Touba Tarvirdizadeh ${ }^{4}$ \\ ${ }^{1}$ Department of Endocrinology, Tabriz University of Medical Sciences, Tabriz, Iran \\ ${ }^{2}$ Department of Surgery, Tabriz University of Medical Sciences, Tabriz, Iran \\ ${ }^{3}$ Department of Pediatrics, Tabriz University of Medical Sciences, Tabriz, Iran \\ ${ }^{4}$ Department of Internal Medicine, Tabriz University of Medical Sciences, Tabriz, Iran
}

Email address:

dr.amir87@gmail.com (A. T. Dereshgi)

\section{To cite this article:}

Taher Manzary, Amir Teimouri Dereshgi, Vahideh Sadra, Ali Jamshidi Fard, Leila Teimouri Dereshgi, Touba Tarvirdizadeh. Thymic Neuroendocrine Tumour Results Cushing's Syndrome. International Journal of Diabetes and Endocrinology.

Vol. 4, No. 4, 2019, pp. 98-103. doi: 10.11648/j.ijde.20190404.12

Received: August 5, 2019; Accepted: November 5, 2019; Published: November 12, 2019

\begin{abstract}
Background: Thymic Neuroendocrine Tumor (TNET) is a rare clinical condition with approximate incidence rate of 2-5\%. Carcinoid tumor of thymus with Cushing's syndrome (CS) is also a rare co-morbid condition. Case information: Here we report a case of a 22-year-old gentleman presented with uncommon features suggestive of CS. He was evaluated and diagnosed with ectopic Adrenocorticotropic hormone (ACTH)-dependent CS due to a TNET. Results: Extensive thymectomy was performed and histopathology confirmed the diagnosis of thymic carcinoma with neuroendocrine differentiation and local and pericardial invasion. Conclusion: Thymic carcinomas may present with symptoms due to mass effect but Cushing syndrome is the most common endocrine manifestation of these tumors. Surgery is the most effective treatment of thymic carcinoma, although chemotherapy and radiotherapy also have been reported to be effective in some cases.
\end{abstract}

Keywords: Cushing's Syndrome, Thymic Carcinoma, Neuroendocrine Tumor

\section{Introduction}

Cushing syndrome (CS), a challenging and rare clinical condition, is a result of prolonged and inappropriate exposure to uncontrolled levels of glucocorticoids. [1]

Typical manifestations of CS include: weight gain with truncal obesity, facial rounding (known as moon face), proximal muscle weakness with significant muscle atrophies specially in extremities, thinning of the skin with purple striae, supraclavicular fat pads and post-cervical fat pads (buffalo hump). Some patients also develop acne, hirsutism and in some women, menstrual irregularity is noticeable. [2]

Thymic neuroendocrine carcinomas are responsible for approximately $2-4 \%$ of all anterior mediastinal tumors [3-5]. Thymic neuroendocrine tumor (TNET) is rare with approximate incidence rate of $2-5 \%$. Among all thymic epithelial tumors, Neuroendocrine tumors (NET) originated from thymus account for only $0.4 \%$. The most common histological type of TNET is atypical carcinoid tumor (ATC) [4].

Thymus neuroendocrine tumors which are associated with Cushing's syndrome can occur at any age between 4 to 64 years, but their peak incidence is between the second and the fourth decades of a patient's life $[1,4]$ as frequently in men as in women $[3,7,8]$.

\section{Case Presentation}

A 22-year-old Azerbaijanis gentleman with no significant past medical history, was referred to our endocrinology clinic for further investigation of hypertension, fatigue, skin darkness and weight gain all started about 6 months ago. He had no history of smoking, alcohol or illicit drug consumption, nor family history of serious physical or mental diseases.

Patient complained that during the preceding 6 months, he 
had difficulties with climbing stairs and gained more than 7 kilograms of weight (body height, $163 \mathrm{~cm}$; current body weight, $60 \mathrm{~kg} ; \mathrm{BMI}, 22.6 \mathrm{~kg} / \mathrm{m}^{2}$ ). On the admission day, he was also complaining of cough that had been lasting for more than a month.

On general assessment, patient was afebrile (37,2 C), His Heart rate was 106 beats/min, respiratory rate was 17 per minute and his blood pressure was $159 / 105 \mathrm{mmHg}$.

Physical examination revealed a mildly round face (figure 1) as well as patchy buccal hyperpigmentation on each buccal mucosa (figure 2), but there were no signs of easy bruising, supraclavicular fat pads, buffalo hump or purple striae.

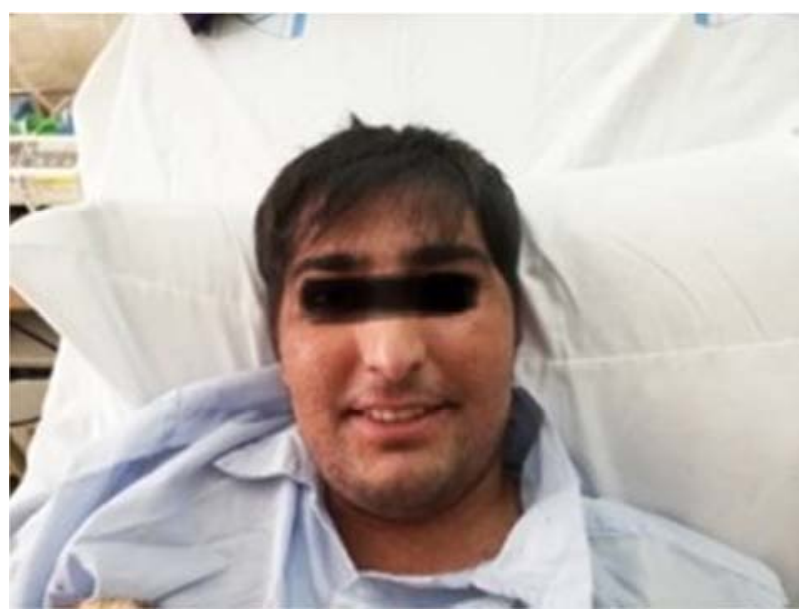

Figure 1. Moon face.

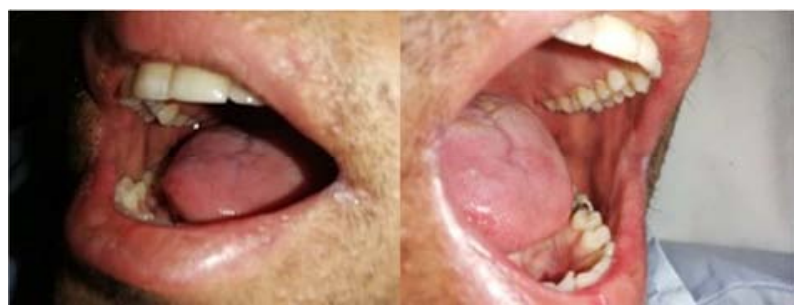

Figure 2. Patchy buccal hyperpigmentation.

There was no organomegaly on abdominal examination as well but, coarse patches of hypertrichosis and erythematous inflammatory papules noticed on the skin of the abdomen (figure 3).

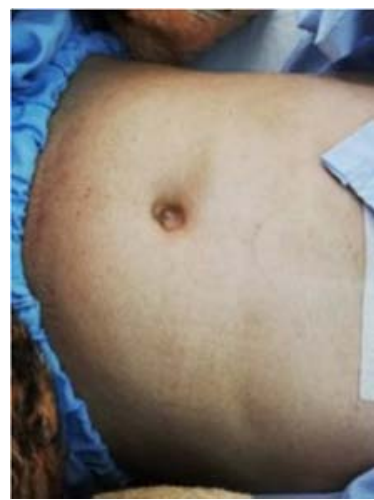

Figure 3. Hypertrichosis and erythematous inflammatory papules.
There was hyperpigmentation on overlying skin of MCP, PIP, DIP joints and nails (body and nail walls) of both hands (figures 4). Also, there was hyperpigmentation on PIP, DIP joints and nails (body and nail walls) of feet (figure 5).

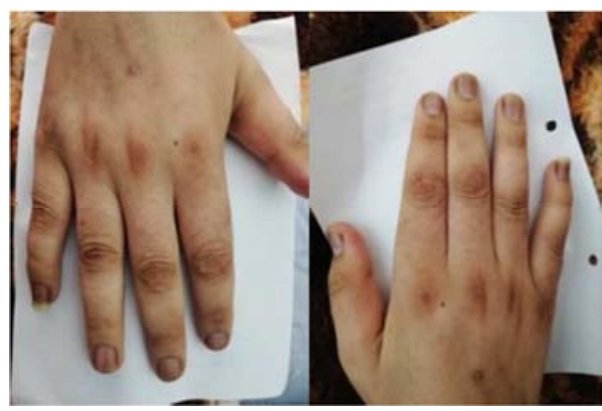

Figure 4. Hyperpigmentation on hand.

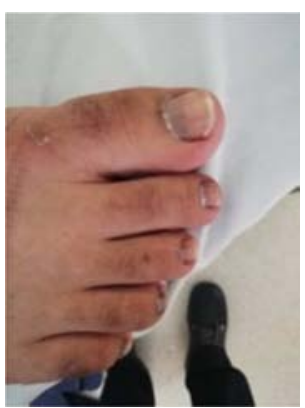

Figure 5. Hyperpigmentation on foot.

Gower's sign also noticed. Muscle forces were decreased symmetrically in both lower extremities (both 3/5) with noticeable decrease in muscle bulk of buttocks and quadriceps areas.

The patient had developmental Tanner stage of P5G5 with normal genitalia (Right testis: $4 \mathrm{~cm}$, Left testis: $4.3 \mathrm{~cm}$ ).

Primary serum serology was within normal range except for $2.9 \mathrm{mmol} / \mathrm{L}$ Potassium (K).

Rest of the physical examination was unremarkable.

\subsection{Investigations}

Cushing's syndrome (CS) suspected based on physical examination. So, serial examinations for CS performed. Initial work up showed morning (8 a.m.) cortisol level of $30.6 \mu \mathrm{g} / \mathrm{dL}(\mathrm{RR}=13-24 \mu \mathrm{g} / \mathrm{dL})$. For further evaluation, overnight Dexamethasone suppression test performed with 1 mg oral Dexamethasone and morning (8 a.m.) serum Cortisol level on the following day measured $35.5 \mu \mathrm{g} / \mathrm{dL} \quad(\mathrm{RR}<1.8$ $\mu \mathrm{g} / \mathrm{dL})$.

Low dose Dexamethasone suppression test performed with $0.5 \mathrm{mg}$ oral dexamethasone every 6 hours for 48 hours and morning (8 a.m.) serum cortisol level measured $27 \mu \mathrm{g} / \mathrm{dL}$ $(\mathrm{RR}<1.8 \mu \mathrm{g} / \mathrm{dL})$.

So, All the results were consistent with the diagnosis of CS.

Also ACTH level revealed $257 \mathrm{pg} / \mathrm{ml} \quad(\mathrm{RR}=10-50$ $\mathrm{pg} / \mathrm{mL}$ ), therefore, High dose Dexamethasone suppression test performed with $8 \mathrm{mg}$ of oral Dexamethasone to find primary source of CS and since no suppression occurred (8 a.m. cortisol level $29.9 \mu \mathrm{g} / \mathrm{dL}$ ) and pituitary protocol 
magnetic resonance imaging (MRI) showed no lesions, diagnoses of ectopic ACTH dependent CS established.

On the next step, for evaluation of the ectopic ACTH source, Imaging studies performed. Multi-slice computed tomography (MSCT) for the chest and abdomen revealed a $3.8-\mathrm{cm}$ mass in anterior mediastinum, in the region of thymus. Also, a 7-cm lung abscess noticed in right lower lobe. (figures 6)
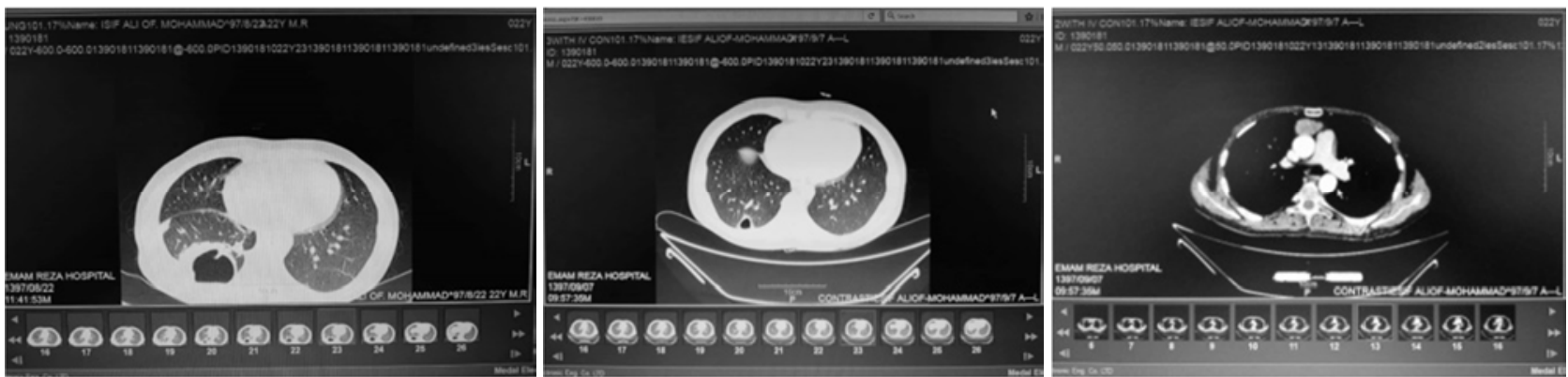

Figure 6. Multi-slice computed tomography showing lung abscess and anterior-mediastinal mass.

Whole body scan and SPECT carried out after intravenous injection of $16.5 \mathrm{mCi}$ Tc-99m- OCT.

A $37.3 * 21.3 \mathrm{~mm}$ focal abnormal anterior mediastinal octreotide avid lesion correlating with findings of MSCT due to ectopic ACTH producing thymus tumor reported. (figures 7)
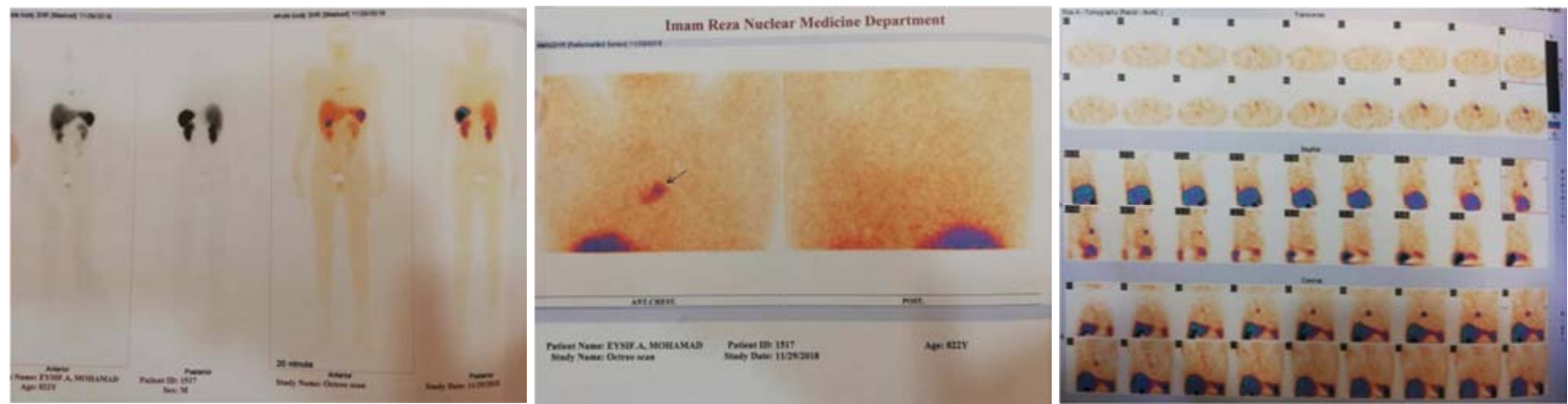

Figures 7. Showing focal abnormal anterior mediastinal octreotide avid lesion after IV injection of $16.5 \mathrm{mCi}$ Tc-99m-OCT.

\subsection{Treatment}

Patient started on $600 \mathrm{mg}$ of intravenous Clindamycin 4 times a day for his lung abscess and $100 \mathrm{mg}$ of Hydrocortisone twice a day prior to operation to prevent post-operative hypotension crisis.

Patient underwent thoracoscopic radical thymectomy. Peroperatively there was a $37-\mathrm{mm}$ diameter hard mass in the right lobe of the thymus infiltrating the pericardium.

The thymus excised radically along with the infiltrated portions of the pericardium and mediastinal lymph nodes. (figure 8)

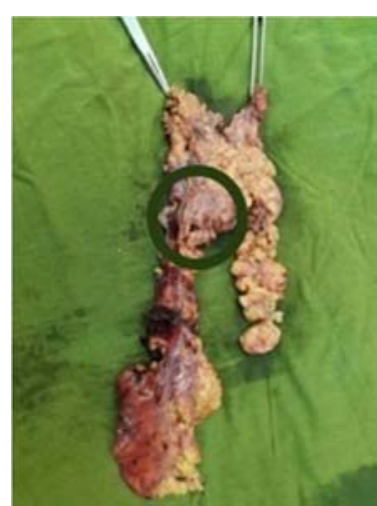

Figure 8. Tumor of thymus.
On the following day, ACTH level dropped to $3 \mathrm{pg} / \mathrm{ml}$.

Pathologic review of the specimen, revealed proliferation of medium sized cells having monomorphic round nuclei with moderate cytoplasm, arranged as nests or sheets with mitotic rate of 2 in $10 \mathrm{HPF}$ and no necrosis.

The neoplastic cells showed positive staining with CK (Figure 9), Chromogranin A (Figure 10) and Synaptophysin but Negative staining with Vimentin, EMa and S100 (Figures 11-13).

The tumor infiltrated surrounding fibroadipose tissue with lymphatic invasion. (Figure 14)

Ki67 was positive in $3-4 \%$ of cells. Diagnosis of Neuroendocrine carcinoma consistent with well differentiated thymic carcinoma established.

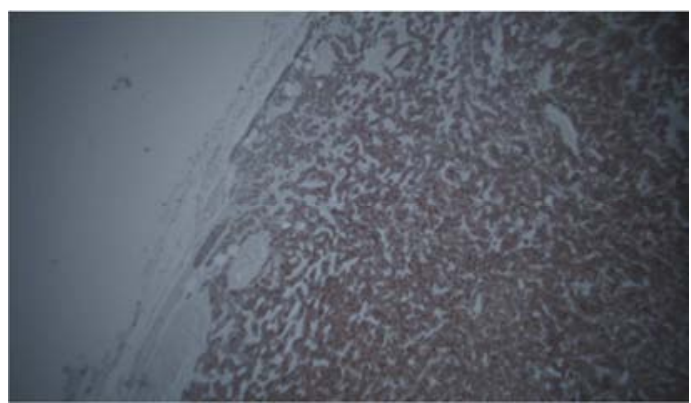

Figure 9. Positive CK staining. 


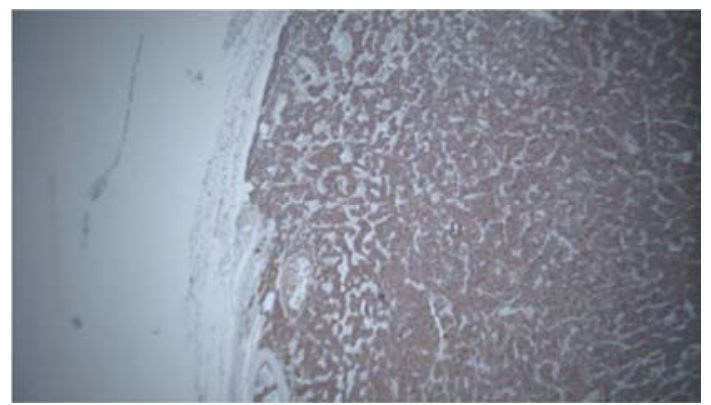

Figure 10. Positive Chromogranin A.

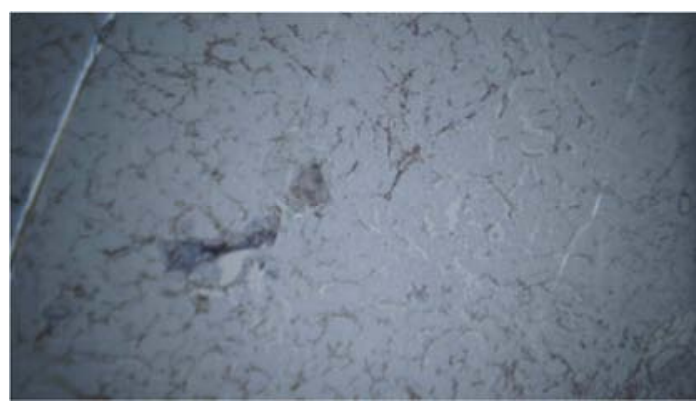

Figure 11. Negative Vimentin.

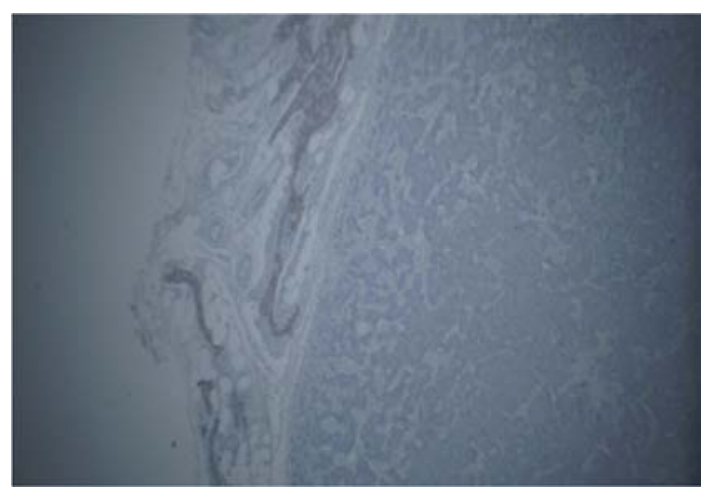

Figure 12. Negative Ema.

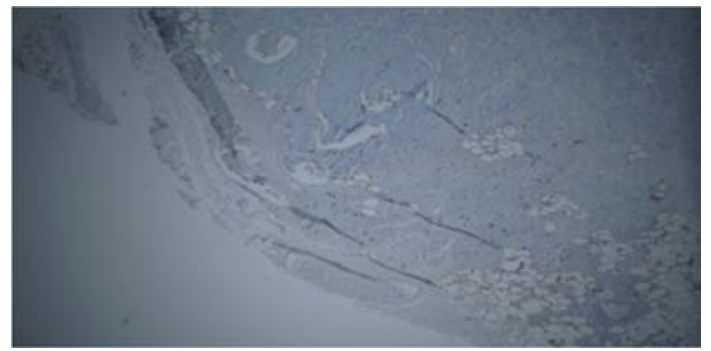

Figure 13. Negative S100.

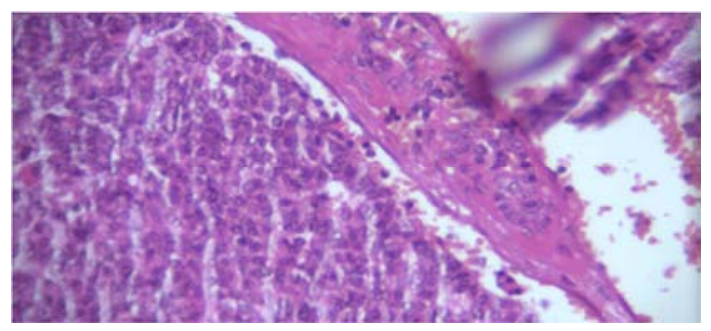

Figure 14. Fibroadipose tissue with lymphatic invasion.

5 days after operation, patient discharged on oral
Prednisolone $(25 \mathrm{mg}$ initially, tapered to $5 \mathrm{mg}$ within a month) and long acting Octreotide.

\section{Outcome and Follow-up}

Six weeks later, patient re-visited in our endocrinology clinic. His BP measured 113/73 mmHg, muscle forces were back to normal (5/5 in both lower and upper limbs) and his serum potassium level was $4.2 \mathrm{mmol} / \mathrm{L}$.

Prednisolone discontinued for 48 hours. Then patient's morning (8 a.m.) serum cortisol level measured $16 \mu \mathrm{g} / \mathrm{dL}$ which was within normal range $(\mathrm{RR}=13-24 \mu \mathrm{g} / \mathrm{dL})$.

Also all his Cushingoid features were completely resolved including buccal, PIP and DIP hyperpigmentation and moon face.

\section{Statement of Ethics}

The authors have no ethical conflicts to disclose. The patient has given his informed consent, including the use of the photographs, imagines and pathology slides.

\section{Discussion}

Cushing's syndrome (CS) is traditionally estimated to affect 10 to 15 people per million populations each year in the United States. [9]

Initially most patients exhibit broad constellation of symptoms, So the diagnosis of CS is often delayed. Also, CS is frequently misdiagnosed by its overlap with more common metabolic conditions such as diabetes mellitus, dyslipidemias, metabolic bone disorders, obesity, hypertension and poly-cystic ovarian syndrome. So, the average interval from beginning of the first symptom to start the treatment is about two years.

Due to the high morbidity and mortality rate associated with prolonged glucocorticoid exposure, including cardiovascular complications, thromboembolic events, immunosuppression and neuro-cognitive disorders, early diagnosis and treatment is of utmost importance. [10-12]

In $80-85 \%$ of cases, endogenous CS is adrenocorticotropic hormone (ACTH) dependent, due to a pituitary or an extrapituitary source (i.e., ectopic ACTH syndrome). In the remaining $10-20 \%$ of cases the disease is ACTH-independent usually the result of an adrenal pathology (gland tumor or adrenal hyperplasia, which can be unilateral or bilateral and benign or malignant). [13, 14]

Ectopic ACTH secreting (EAS) tumors - constitute $15 \%$ of Cushing syndromes - usually present with rapid onset disease and is almost always difficult to diagnose and treat, especially in terms of localization of the ectopic source.

EAS is most commonly associated with lung cancer (mainly small cell lung cancer), bronchial carcinomas, thymic and pancreatic carcinoids. Medullary thyroid cancer, small cell colon or neuroendocrine tumors (NET) of unidentified source, or pheochromocytomas account for the remaining published cases of EAS. 
The relationship between a thymic tumor and EAS was first described in 1931 by Leyton et al and since then, there has been a modest but increasing number of literature describing this correlation. [15-17]

Thymic carcinoma is a rare aggressive neoplasm and thymic neuroendocrine carcinoma accounts for approximately $2-4 \%$ of all anterior mediastinal tumors.

Thymic Carcinoid Tumors which are originated from the Argynophil cells in the normal thymus are divided in three groups: typical carcinoid, atypical carcinoid and small-cell carcinoma of the thymus.

Thymus neuroendocrine tumors (TNET) manifesting as Cushing's syndrome can occur at any age from 4 to 64 years, but their peak prevalence is between the second and the fourth decades of a human's life [3,6] as frequently in men as in women $[3,7,8]$.

TNET may present with symptoms due to mass effect including hoarseness, superior vena cava syndrome, phrenic nerve paralysis, chest pain, cough and shortness of breath or with endocrinopathy [3, 4].

Cushing syndrome is the most common endocrine manifestation of these tumors [8]. There have subsequently emerged case reports of 92 patients and a case series of 12 patients describing TNETs as the cause of Cushing's syndrome over 25 years by Neary et al. [18-22]

The most common histological type of TNET reported to be atypical carcinoids tumors. Under the 2015 WHO grading scheme, NETs of the lungs and thymus fall into one of the following three grades [23]:

a. Low-grade tumors, $<2$ mitoses per 10 high power fields (HPFs; or $2 \mathrm{~mm} \mathrm{2)}$ and no necrosis

b. Intermediate tumors, $2-10$ mitoses per $10 \mathrm{HPFs} / 2 \mathrm{~mm} 2$ and/or foci of necrosis

c. High-grade tumors, $>10$ mitoses per $10 \mathrm{HPFs} / 2 \mathrm{~mm} 2$ Travis WD

Precise pretherapeutic diagnosis of CS is important and must be confirmed by increased cortisol level in the serum or in the 24 hours urine collection (Urine free Cortisol-UFC), which must also remain elevated despite a low dose dexamethasone suppression test $(0.5 \mathrm{mg}$ oral dexamethasone every 6 hours for 48 hours) $[3,6]$. The next step in evaluating a Cushing patient is measuring serum ACTH levels. The presence of high a ACTH level in a Cushing patient excludes the possibility of an autonomous secretion of cortisol by an adrenal tumor [27].

Radiographic and nuclear imaging studies both play significant roles in terms of localization and management of all carcinoid tumors [8, 27]. Spiral computed tomography (Spiral CT scan), magnetic resonance imaging (MRI) and ultrasonography are used for determining the precise location of tumors and also in some cases for following up the response to the treatment [3]. Octreotide scan can determine early advanced disease which other diagnostic procedures were not able to reveal $[3,8]$.

Surgery is the most important part of the treatment of thymic carcinoma, although chemotherapy and radiotherapy also have been reported to be effective in some cases (except for widely metastatic tumors) [3, 4]. In the first year after surgery, patients should be monitored with serum and urinary free cortisol (UFC) testing as well as conventional imaging.

As these tumors express somatostatin receptors, 90YDOTA0Tyr3 octreotide and 177Lu-DOTATATE therapies are considered as useful treatment regimens. There is also a straight correlation between response to therapy and the intensity of the uptake by tumor.

The most common used chemotherapy drugs for thymic carcinoma are platinum based regimens. However chemotherapy do not significantly increase survival in these patient. Nakamura et al found no association between histological subtypes and prognosis in patients with advanced thymic carcinoma who received chemotherapy and indicated that platinum based chemotherapy is only marginally effective for advanced thymic carcinoma [28]. Suster and Rosai also found no beneficial effect in chemotherapy. The prognosis is reported a five years survival of approximately $50 \%$ for patients with well-defined thymic carcinoid tumor and $27 \%$ for those with moderately differentiated thymic carcinoid tumors [29]. Incomplete resection, tumor extension, distant metastasis and high histological grade are factors of a poor prognosis.

All patients should have an baseline octreotide scan and If a baseline scan is positive, an octreotide scan is recommended either once a year or earlier if evidence of disease is seen [24, 28].

Five-year survival rate is estimated about of $27 \%$. Most patients present with local recurrence or metastasis within 5 years after surgery and die within 10 years $[3,25,26]$.

\section{Conclusion}

Cushing syndrome is the most common endocrine manifestation of Thymic Neuroendocrine tumors. Surgery is the most important part of treatment but chemotherapy and radiotherapy should also be considered in metastatic disease.

\section{References}

[1] Newell-Price J Bertagna X Grossman A B Nieman L K 2006 Cushing's syndrome. Lancet 367 1605-1617. (doi: 10.1016/S0140-6736(06)68699-6).

[2] Lacroix A, Feelders RA, Stratakis CA, et al. Cushing's syndrome. Lancet. 2015 Aug 29; 386 (9996): 913-27.

[3] Shields TW, LoCicero JI, Reed CE, Feins RH. General thoracic surgery. 7th edn: Lippincott Williams \& Wilkins. 2009.

[4] Ose N, Maeda H, Inoue M, Morii E, Shintani Y, Matsui H, et al. Results of treatment for thymic neuroendocrine tumours: multicentreclinicopathological study. Interact Cardiovasc Thorac Surg. 2018; 26: 18-24.

[5] Aghajanzadeh M, Alavi A, Aghajanzadeh G, Massahania S. Stiff man syndrome with invasive thymic carcinoma. Arch Iran Med. 2013; 16: 195-156. 
[6] de Perrot M, Spiliopoulos A, Fischer S, Totsch M, Keshavjee S. Neuroendocrine carcinoma (carcinoid) of the thymus associated with Cushing's syndrome. Ann Thor Surg. 2002; 73: 675-681.

[7] Aghajanzadeh M, Alavy A, Hoda S, Mohammadi F. Carcinoid tumor of lung with Cushing's syndrome. Arch Iran Med. 2007; 10: 94-6.

[8] Fernandez-Fernandez F, Halperin I, Manzanares J, Flores L, Lomena F, Vilardell E. Localization and postoperative followup of a bronchial carcinoid tumor causing Cushing's syndrome by 111 In-DTPA labeled octreotide scintigraphy. J Endocrinol Invest. 1997; 20: 327-330.

[9] S. Sharma, L. K. Nieman, R. A. Feelders. Clin Epidemiol, 7 (2015), pp. 281-293.

[10] Psychiatric aspects of Cushing's syndrome. Kelly WF QJM. $1996 \mathrm{Jul} ; 89$ (7): 543-51.

[11] Broder MS, Neary MP, Chang E, et al: Incidence of Cushing's syndrome and Cushing's disease in commercially-insured patients $<65$ years old in the United States. Pituitary 2015; 18: 283-289.

[12] A thymic neuroendocrine tumor in a young female: a rare cause of relapsing and remitting Cushing's syndrome in Endocrinology, Diabetes \& Metabolism Case Reports, Authors: M J Trott 1, G Farah 1, V J Stokes 1, L M Wang 1 and A B Grossman 1, volume 2016, issue 1.

[13] Guaraldi F, Salvatori R J Am Board Fam Med. 2012 Mar-Apr; 25 (2): 199-208.

[14] Bansal V, Asmar N, Selman W, Arafah B. Pitfalls in the Diagnosis and Management of Cushing's Syndrome. Neurosurg Focus. 2015; 38: 1-11.

[15] Ilias I Torpy D J Pacak K Mullen N Wesley R A Nieman L K 2005 Cushing's syndrome due to ectopic corticotropin secretion: twenty years' experience at the National Institutes of Health. Journal of Clinical Endocrinology and Metabolism 90 4955-4962. (doi: 10.1210/jc.2004-2527).

[16] Isidori A M Kaltsas G A Pozza C Frajese V Newel-Price J Reznek D H Jenkins P J Monson J P Grossman A B Besser G M 2006 The ectopic adrenocorticotropin clinical features, diagnosis, management, and long-term follow up. Journal of Clinical Endocrinology and Metabolism 91 371-377. (doi: 10.1210/jc.2005-1542).

[17] Leyton O Turnbull H M Bratton A B 1931 Primary cancer of the thymus with pluriglandular disturbance. Journal of Pathology and Bacteriology 34 635-660. (doi: 10.1002/(ISSN)1555-2039).

[18] Multidisciplinary treatment of advanced thymic neuroendocrine carcinoma (carcinoid): report of a successful case and review of the literature. Filosso PL, Actis Dato GM, Ruffini E, Bretti S, Ozzello F, Mancuso M. J Thorac Cardiovasc Surg. 2004 Apr; 127 (4): 1215-9.
[19] Neuroendocrine carcinoma (carcinoid) of the thymus associated with Cushing's syndrome. de Perrot M, Spiliopoulos A, Fischer S, Totsch M, Keshavjee S. Ann Thorac Surg. 2002 Feb; 73 (2): 675-81.

[20] Amin MB, Edge SB, Greene FL, Byrd DR, Brookland RK, Washington MK, eds. AJCC Cancer Staging Manual. 8th ed. New York: Springer; 2017.

[21] Neary NM, Lopez Chavez A Abel B S Boyce A M Schaub N Kwong K Stratakis C A Moran C A Giaccone G Nieman L K 2012 Neuroendocrine ACTH-producing tumor of the thymus - experience with 12 patients over 25 years. Journal of Clinical Endocrinology and Metabolism 97 2223-2230. (doi: 10.1210/jc.2011-3355).

[22] Syncope in a patient with a large left ventricular hydatid cyst: An unusual presentation, Beheshtirouy, Samad et al. International Journal of Cardiology, Volume 172, Issue 3, e385-e386.

[23] Brambilla E, Nicholson AG, Yatabe Y, Austin JH, Beasley MB, et al. The 2015 World Health Organization Classification of Lung Tumors: Impact of Genetic, Clinical and Radiologic Advances Since the 2004 Classification. J Thorac Oncol. 2015 Sep. 10 (9): 1243-60.

[24] Survival and response after peptide receptor radionuclide therapy with [90Y-DOTA0, Tyr3] octreotide in patients with advanced gastroenteropancreatic neuroendocrine tumors. Valkema R, Pauwels S, Kvols LK, Barone R, Jamar F, Bakker WH, Kwekkeboom DJ, Bouterfa H, Krenning EP. Semin Nucl Med. 2006 Apr; 36 (2): 147-56.

[25] Peptide receptor radionuclide therapy with $177 \mathrm{Lu}$-octreotate in patients with foregut carcinoid tumours of bronchial, gastric and thymic origin. van Essen M, Krenning EP, Bakker WH, de Herder WW, van Aken MO, Kwekkeboom DJ. Eur J Nucl Med Mol Imaging. 2007 Aug; 34 (8): 1219-27.

[26] Primary neuroendocrine tumors of the thymus. Chaer R, Massad MG, Evans A, Snow NJ, Geha AS. Ann Thorac Surg. 2002 Nov; 74 (5): 1733-40.

[27] Maroun J, Kocha W, Kvols L, Bjarnason G, Chen E, Germond $\mathrm{C}$, et al. Guidelines for the diagnosis and management of carcinoid tumours. Part 1: the gastrointestinal tract. A statement from a Canadian National Carcinoid Expert Group. Curr Oncol. 2006; 13: 67.

[28] Nakamura Y, Kunitoh H, Kubota K, Sekine I, Shinkai T, Tamura T, et al. Platinum-based chemotherapy with or without thoracic radiation therapy in patients with unresectable thymic carcinoma. Jpn J Clin Oncol. 2000; 30: 385-388.

[29] Moran CA, Suster S. Primary neuroendocrine carcinoma (thymic carcinoid) of the thymus with prominent oncocytic features: a clinicopathologic study of 22 cases. Mod Pathol. 2000; 13: 489. 